\title{
Inventário de Habilidades Sociais para Cuidadores de Idosos (IHS-CI): evidências de validade
}

\author{
Francine Náthalie Ferraresi Rodrigues Queluz ${ }^{1}$ \\ Universidade São Francisco, Campinas-SP, Brasil \\ Elizabeth Joan Barham, Zilda Aparecida Pereira Del Prette \\ Universidade Federal de São Carlos, São Carlos-SP, Brasil \\ Anne Marie Germaine Victoria Fontaine \\ Universidade do Porto, Porto, Portugal \\ Fabian Orlando Olaz \\ Universidade Nacional de Córdoba, Córdoba, Argentina
}

\section{RESUMO}

Cuidar de um idoso dependente requer aprender informações e desenvolver habilidades, incluindo as habilidades sociais. Faz-se necessário, então, um instrumento para medir essas habilidades de cuidadores de idosos familiares. O objetivo geral do presente trabalho foi construir o Inventário de Habilidades Sociais para Cuidadores familiares de Idosos (IHS-CI). Foram realizados dois estudos: (1) avaliação semântica e de conteúdo do IHS-CI e (2) análise de evidências da estrutura interna e precisão do IHS-CI. No Estudo 1, especialistas aprovaram 31 itens da versão inicial do instrumento. No Estudo 2, o IHS-CI foi aplicado em 205 cuidadores. Com base na análise fatorial exploratória, a melhor estrutura foi: expressividade emocional $(\alpha=0,87)$, comunicação assertiva $(\alpha=0,79)$ e busca por informação $(\alpha=0,60)$. A confiabilidade interna global do instrumento $(\alpha=0,89)$ foi excelente. Ainda são necessários estudos para ampliar as evidências de validade do IHS-CI.

Palavras-chave: habilidades sociais; cuidadores; idosos; estresse; avaliação psicológica.

\section{ABSTRACT - Social Skills Inventory for Caregivers of Elderly People (IHS CI): Evidence of validity}

Caring for a dependent elderly relative requires learning information and developing skills, including social skills. As such, an instrument for measuring social skills of family caregivers is needed. The aim of this research was to construct a Social Skills Inventory for family Caregivers of the Elderly (IHS CI). Two studies were conducted: (1) a semantic and content analysis of the IHS IC and (2) an analysis of the internal structure and precision of the IHS CI. In Study 1, experts approved 31 items of the initial version of the instrument. In Study 2 , the IHS CI was answered by 205 caregivers. Based on an exploratory factor analysis, the best structure was: emotional expressiveness $(\alpha=0,87)$, assertive communication $(\alpha=0,79)$ and information seeking $(\alpha=0,60)$. The internal reliability of the instrument was excellent $(\alpha=0,89)$. Further studies are needed, in order to obtain additional evidence about the validity of the IHS CI.

Keywords: social skills; caregivers; elderly; stress; psychological evaluation.

RESUMEN - Inventario de Habilidades Sociales para cuidadores de ancianos (IHS-CI): validez de evidencia

Cuidar de un anciano dependiente requiere aprender información y desarrollar habilidades, incluyendo las habilidades sociales. Por esto, es necesario tener un instrumento para medir esas habilidades de cuidadores de ancianos familiares. El objetivo de este estudio fue construir el Inventario de Habilidades Sociales para Cuidadores familiares de ancianos (IHS-CI). Se realizaron dos estudios: (1) evaluación semántica y de contenido de los ítems del IHS-IC y (2) análisis de la evidencia de la estructura interna y precisión del IHS-CI. En el Estudio 1, expertos aprobaron 31 artículos de la versión original del instrumento. En el estudio 2, se aplicó el IHS-IC a una muestra de 205 cuidadores de ancianos. Basado en el análisis factorial exploratorio, la mejor estructura fue: expresividad emocional $(\alpha=0,87)$, comunicación asertiva $(\alpha=0,79)$ y búsqueda de información $(\alpha=0,60)$. La fiabilidad interna general del instrumento fue excelente $(\alpha=0,89)$. Se necesitan más estudios para aportar mayor evidencia de validez del IHS-CI.

Palabras clave: habilidades sociales; cuidadores; ancianos; estrés; evaluación psicológica.

Com a elevação da expectativa de vida e o subsequente aumento na porcentagem de pessoas com doenças crônico-degenerativas, a tarefa de cuidar de um idoso próximo torna-se cada vez mais comum. Os cuidadores de idosos normalmente são do sexo feminino, cônjuges ou filhas do idoso dependente, estão na meia-idade e têm baixo poder 
aquisitivo (Gratão et al., 2013; Novelli, Nitrini, \& Caramelli, 2010; Wang, Robinson, \& Carter-Harris, 2014). Neste contexto, percebe-se que as pessoas idosas cuidadas geralmente apresentam múltiplos problemas de saúde, que interferem na sua funcionalidade em mais de uma esfera, de forma que os cuidadores têm que lidar com um contexto caracterizado por limitadas opções de atividades e altas demandas de assistência (Lorenzini, 2014). A dificuldade para se adaptar e para lidar com essas demandas, com o tempo, pode gerar estresse e sentimentos de sobrecarga no cuidador (Barham, Pinto, Andrade, Lorenzini, \& Ferreira, 2015; Li, Cooper, Bradley, Shulman, \& Ryan, 2012; Lin, Chen, \& Li, 2013).

Estudos indicam que pessoas que cuidam de um idoso dependente por muito tempo tendem a apresentar um aumento nos níveis de estresse, diminuição na qualidade de vida e maior probabilidade de se sentirem deprimidos (Lin et al., 2013; Pereira \& Carvalho, 2012; Tomomitsu, Perracini, \& Neri, 2014). Todos esses problemas podem acarretar, para o cuidador, dificuldade em gerenciar relacionamentos com as diferentes pessoas que fazem parte de sua rotina, resultando em uma carga emocional pesada. Pessoas com habilidades sociais (HS) bem desenvolvidas conseguem conversar sobre como realizar atividades para suprir as necessidades e os interesses dos envolvidos no contexto de cuidado, de forma a se apoiarem em seu círculo social para lidar com os desafios do processo de envelhecimento (Pinto, Barham, \& Del Prette, 2016). Quando as necessidades de alguém — seja do idoso ou do cuidador — são ignoradas, surge uma situação de negligência do idoso ou de exploração do cuidador (Pinto, Barham, \& Albuquerque, 2013). Nesse sentido, é importante que o cuidador desenvolva suas HS dentro do contexto de cuidado do idoso, o que poderia ajudá-lo a se manter em um melhor estado de bem-estar. No entanto, pesquisas sobre HS em cuidadores de idosos são muito escassas (Pinto et al., 2016).

\section{Habilidades Sociais}

Del Prette e Del Prette (2013) consideram qualquer comportamento, ou sequência de comportamentos, em uma situação social como "desempenho social", que pode ser considerado como socialmente competente ou não. Assim, o conceito de "competência social" envolve uma avaliação ou julgamento a respeito da adequação do desempenho de uma pessoa e do efeito que produz em uma determinada situação, enquanto que o conceito de HS envolve as classes de comportamento que existem no repertório de um indivíduo e que são requeridas para um melhor desempenho social (Del Prette \& Del Prette, 2013). É importante lembrar que a competência social envolve usar essas habilidades para atender às necessidades ou interesses das pessoas presentes em um contexto específico. Assim, uma pessoa socialmente competente em um contexto conjugal ou profissional, por exemplo, pode não ser competente no contexto de cuidar de um familiar idoso, no qual há outras demandas e a necessidade de estratégias para conciliar diferenças de crenças e expectativas sobre como organizar rotinas e lidar com as limitações de saúde do idoso. Com base na análise da estrutura interna de seu instrumento de HS para adultos universitários, Del Prette e Del Prette (2001) propuseram cinco classes de HS: enfrentamento e autoafirmação; expressão de sentimentos positivos; conversação e desenvoltura social; autoexposição a desconhecidos e situações novas; e autocontrole da agressividade.

Posteriormente, considerando as demandas comumente encontradas na prática do treinamento das HS, Del Prette e Del Prette (2013) propuseram um arranjo de classes de HS relevantes para adultos em geral, que deveriam incluir: HS de comunicação, HS de civilidade, HS assertivas e para exercer direitos e cidadania, HS empáticas, HS de trabalho em grupo e HS de expressar sentimentos positivos. Entende-se que o desenvolvimento de HS apropriadas ao contexto de cuidar de um idoso dependente é importante, pois pessoas capazes de manter relações positivas e de apoio mútuo podem manejar ou prevenir reações negativas quando surgem novos desafios (Muela, Torres, \& Peláez, 2001; Pinto \& Barham, 2014a; 2014b). Como resultado, os cuidadores não interpretariam as demandas desse papel como fontes de estresse e sobrecarga. Desta forma, estudar as especificidades de HS entre cuidadores de idosos se mostra pertinente, uma vez que os estudos existentes na literatura indicam que um desempenho socialmente competente implica em maior autoestima e qualidade de vida, melhores relacionamentos interpessoais e também em um menor índice de depressão, de sobrecarga e de percepção de conflitos familiares (Barham et al., 2015; Faleiros, 2009; Muela et al., 2001; Pinto \& Barham, 2014b; Robinson, 1988; 1990; Robinso n \& Yates, 1994). Pode-se esperar que indivíduos socialmente competentes encontrem maneiras de conciliar as necessidades das pessoas do seu convívio, desenvolvam relacionamentos interpessoais de maior qualidade, sejam menos propensos a doenças e mais saudáveis, de maneira geral (Del Prette \& Del Prette, 2010; 2013).

Dada a importância de identificar déficits e recursos em HS ligados ao contexto, ainda pouco estudado, de cuidar de um idoso dependente - uma situação enfrentada por uma proporção cada vez maior da população brasileira -, defende-se a necessidade de construção de instrumentos para isso. Especialmente quando alguém é novato no papel de cuidador de idoso, pode ser de fundamental importância receber ajuda para descobrir como oferecer cuidado de boa qualidade, e ao mesmo tempo cuidar de si. Aferir com maior precisão o repertório de HS de cuidadores, nesse contexto específico, poderia ser particularmente útil para delinear intervenções que tenham como objetivo manter ou melhorar a qualidade de vida desse cuidador, o que certamente contribuiria também para melhorar sua inserção social, concomitantemente com as condições de vida do idoso sob seus cuidados. 


\section{Avaliação de habilidades sociais}

Del Prette e Del Prette (2010) descreveram os instrumentos já publicados na literatura nacional para avaliação de HS em diferentes contextos. Ao todo, foram descritos 12 instrumentos, dentre os quais 7 tinham crianças e adolescentes como população-alvo, 4 tinham adultos como população-alvo e 1 era voltado para casais. Com base nessas informações, em conjunto com uma busca na literatura internacional de instrumentos em HS voltados ao contexto de cuidado de um idoso dependente, foi encontrado um instrumento desenvolvido por Franzmann, Krause, Haberstroh e Pantel (2014), no idioma alemão, para avaliação da competência social em cuidadores de idosos - sendo que esse instrumento foi construído e validado para cuidadores formais que trabalham em asilos, enfocando relações profissionais entre cuidadores e idosos. Pode-se constatar, assim, que as medidas construídas para a avaliação de HS, presentes na literatura acadêmica, permitem investigar as HS em vários contextos, mas ainda não entre cuidadores familiares de idosos (Bender \& Calvetti, 2015). Dado o caráter situacional das HS (Del Prette \& Del Prette, 2013), as escalas existentes no Brasil, para a avaliação destas, não são suficientemente pertinentes ao contexto de cuidado de um idoso. Assim, não permitem identificar cuidadores com déficits das HS requeridas nesse contexto, nem aferir melhoras no repertório social do cuidador, decorrentes de um treinamento direcionado para desenvolver tais habilidades deficitárias. O Inventário de Habilidades Sociais (IHS-Del Prette), embora seja um instrumento de medida de HS gerais, foi construído e normatizado para estudantes universitários, podendo ser estendido, no máximo, para adultos jovens (Del Prette \& Del Prette, 2001; 2013). Portanto, pode-se afirmar que há uma lacuna de conhecimento nessa área e uma necessidade de construção de um instrumento específico para avaliar as HS de cuidadores de idosos, inclusive como forma de subsidiar o planejamento e a avaliação de intervenções. A aplicação de um instrumento dessa natureza permitiria aferir mudanças confiáveis e clinicamente significativas nessa população.

Diante do exposto, o objetivo do presente estudo foi construir um Inventário de Habilidades Sociais para Cuidadores familiares de Idosos (IHS-CI). Esse objetivo foi abordado por meio de dois estudos: um para a avaliação semântica e de conteúdo do instrumento (Estudo 1), e outro para obter evidências em relação à estrutura interna e de precisão do IHS-CI (Estudo 2).

\section{Aspectos éticos}

O projeto foi aprovado pelo Comitê de Ética da Universidade Federal de São Carlos (Parecer número 144.507/2012). Os procedimentos do presente estudo respeitaram o constante na Declaração de Helsinki. Todos os participantes receberam informações acerca dos objetivos da pesquisa, das atividades a serem desenvolvidas e de seus direitos, antes de assinarem o Termo de Consentimento Livre e Esclarecido. A identidade de todos os participantes foi mantida em sigilo.

\section{Estudo 1}

\section{Evidência de validade baseada no conteúdo do Inventário de Habilidades Sociais para Cuidadores familiares de Idosos}

Uma primeira versão do Inventário de Habilidades Sociais de Cuidadores Familiares de Idosos (IHS-CI), com 37 itens, foi construída com base em informações obtidas por meio de:

1. uma revisão da literatura sobre o uso de HS entre cuidadores de idosos; e

2. entrevista com 3 tipos de informantes (50 cuidadores, 25 idosos dependentes e lúcidos e 25 profissionais da área de gerontologia) a respeito de HS que consideravam importantes em cuidadores familiares de idosos.

A entrevista respondida pelos participantes continha questões elaboradas com base nos fatores do IHS-Del Prette (Del Prette \& Del Prette, 2001), sendo elas: enfrentamento e autoafirmação; autoafirmação na expressão de sentimentos positivos; conversação e desenvoltura social; autoexposição a desconhecidos e situações novas; e autocontrole da agressividade. Uma breve definição de cada dimensão foi incluída para ajudar na compreensão de cada classe de habilidade por parte dos entrevistados e, em seguida, foi solicitado aos participantes que dessem exemplos de situações de seu dia a dia dentro de cada classe de HS. Ao final da entrevista, havia uma parte aberta, na qual foi solicitado aos respondentes que dessem uma nota de 0 a 10 para cada item do IHS-Del Prette, considerando sua importância no contexto de cuidado de um idoso dependente. Os itens que obtiveram nível de concordância maior ou igual a $80 \%$ permaneceram no instrumento (Pasquali, 1996). Dos 38 itens do IHS-Del Prette, 29 foram mantidos, mas adaptados para retratar interações interpessoais ligadas ao contexto de cuidado de um idoso dependente. Ao final desse processo, foram elaborados oito itens adicionais, com base em interações interpessoais importantes para cuidadores de idosos identificados na literatura acadêmica ou nas entrevistas.

\section{Método}

\section{Participantes}

Participaram do presente estudo:

1. Cinco juízes com formação em psicologia, sendo três doutores e dois doutorandos que possuíam conhecimentos avançados nas áreas de psicometria e de HS na primeira etapa do presente estudo; e

2. Vinte cuidadores familiares de idosos (16 do sexo feminino e 4 do sexo masculino, com idade média 
de 50 anos, variando entre 25 e 73 anos, de diferentes níveis socioeconômicos), para verificar problemas adicionais de clareza e de compreensão dos itens do IHS-CI após a avaliação dos especialistas.

\section{Instrumento}

A primeira versão do IHS-CI continha 37 itens, 10 de autoafirmação na expressão de sentimentos positivos, 10 de conversação e desenvoltura social, 7 de enfrentamento e autoafirmação, 6 de autocontrole da agressividade e 4 de autoexposição a desconhecidos e situações novas. Os cuidadores deveriam responder sobre a frequência com que apresentam o comportamento descrito em cada item, em escala de "nunca", "de vez em quando", "muitas vezes" ou "sempre".

\section{Procedimento}

Foi solicitado aos juízes que avaliassem a consistência semântica e de conteúdo dos itens (Cozby, 2003; Pasquali, 2007; Tornimbeni, Pérez, \& Olaz, 2008). Para tanto, eles deveriam apontar se estavam de acordo ou não com o conteúdo de cada um dos itens, o nível de qualidade formal do item em uma escala de pontuação de 1 a 5 e, caso considerassem pertinente, fazer observações sobre cada item avaliado. Com base nas respostas dos juízes, foi calculado o nível de concordância em relação ao conteúdo dos itens, por meio do coeficiente de validade de conteúdo V de Aiken (Aiken, 1980; 1985). Os itens que obtiveram nível de concordância maior ou igual a $80 \%$ permaneceram no instrumento (Pasquali, 1996). Em seguida, 20 cuidadores familiares de idosos que participavam de um programa de atendimento a idosos na cidade de São Carlos, São Paulo, foram convidados a participar do estudo. Após o aceite do convite, a pesquisadora combinou com os cuidadores um local que lhes fosse conveniente para a realização da pesquisa. Após essa etapa, o instrumento - já avaliado pelos especialistas com 31 itens foi aplicado para avaliar possíveis problemas adicionais de clareza e de compreensão dos itens.

\section{Resultados}

Após a avaliação dos especialistas, 31 itens permaneceram no instrumento. $\mathrm{Na}$ etapa seguinte, o instrumento foi aplicado em cuidadores, com três itens modificados para se adequarem à população com baixa escolaridade. A modificação foi realizada para trocar a expressão "exponho" por "digo", ex: "exponho o que penso" por "digo o que penso".

\section{Estudo 2}

\section{Análise fatorial exploratória do instrumento}

No Estudo 2, foi realizada a análise fatorial exploratória (AFE), visando identificar, dentro do campo das HS, como os itens do instrumento iriam se agrupar.

\section{Método}

\section{Participantes}

Participaram do estudo 205 cuidadores de idosos familiares, com idade média de 51 anos $(D P=14)$, variando entre 18 e 87 anos. Em média, eles exerciam essa tarefa há 5 anos $(D P=4,7)$. Os cuidadores foram recrutados junto ao Serviço de Atendimento Domiciliar (SAD) das cidades de São Carlos e de Campinas, na Unidade Saúde Escola da Universidade Federal de São Carlos, e por indicação de outros participantes ou de pessoas conhecidas pelas pesquisadoras, nas cidades de São Paulo e de Itapira, todas no estado de São Paulo. Como critério de inclusão, os participantes teriam que ser maiores de 18 anos e familiares dos idosos que cuidavam. Cuidadores formais ou pagos foram excluídos dessa amostra. A coleta de dados foi realizada nas residências dos mesmos ou em algum lugar escolhido por eles, garantindo o sigilo necessário. Os dados sociodemográficos são apresentados na Tabela 1.

\section{Instrumentos}

\section{Características Sociodemográficas}

Um questionário foi elaborado para verificar as características sociodemográficas (sexo, idade, escolaridade, estado civil, entre outros) dos participantes, visando descrever a amostra obtida.

\section{Critério de Classificação Econômica Brasil}

Este instrumento permite avaliar o nível socioeconômico das famílias com base no poder aquisitivo, no número de bens de consumo duráveis, na presença de empregada doméstica e no grau de instrução do chefe da família, dividindo a população em sete classes (A, B1, B2, C1, C2, D e E) (ABEP, 2013).

\section{Inventário de Habilidades Sociais}

para Cuidadores Familiares de Idosos

O IHS-CI com 31 itens foi aplicado. Para respondê-lo, os participantes deveriam indicar a frequência com que apresentavam o comportamento descrito em cada item - "nunca" (pontuação 1), "de vez em quando" (pontuação 2), "muitas vezes" (pontuação 3) ou "sempre" (pontuação 4). Quanto maior a pontuação do participante, melhor o seu repertório de HS relatado.

\section{Procedimento}

Após contato com representantes da Secretaria de Saúde da Prefeitura Municipal de São Carlos e de Campinas, e com as Unidades de Atendimento ao Idoso - para indicação de cuidadores com o perfil para este estudo - a pesquisadora convidou cada um dos cuidadores, por telefone, para participar da pesquisa. Para os que aceitaram (todos exceto cinco), 
foi agendado um horário e escolhido um local que fosse conveniente para aplicação dos instrumentos, o que foi precedido pela assinatura do Termo de Consentimento Livre e Esclarecido.

Tabela 1

Perfil sociodemográfico dos participantes

\begin{tabular}{|c|c|c|}
\hline Variável & $n$ & $\%$ \\
\hline \multicolumn{3}{|l|}{ Sexo } \\
\hline Feminino & 180 & 87,8 \\
\hline Masculino & 25 & 12,2 \\
\hline \multicolumn{3}{|l|}{ Faixa etária (anos) } \\
\hline $18-30$ & 24 & 11,7 \\
\hline $31-50$ & 58 & 28,3 \\
\hline $51-70$ & 109 & 53,2 \\
\hline Acima de 70 & 14 & 6,8 \\
\hline \multicolumn{3}{|l|}{ Estado civil } \\
\hline Solteiro & 59 & 28,8 \\
\hline Casado/união estável & 105 & 51,2 \\
\hline Separado & 27 & 13,2 \\
\hline Viúvo & 14 & 6,8 \\
\hline \multicolumn{3}{|l|}{ Escolaridade } \\
\hline $\begin{array}{l}\text { Analfabeto ou } \\
\text { ensino fundamental I incompleto }\end{array}$ & 19 & 9,3 \\
\hline $\begin{array}{l}\text { Ensino fundamental I } \\
\text { ou II incompleto }\end{array}$ & 36 & 17,6 \\
\hline $\begin{array}{l}\text { Ensino fundamental II } \\
\text { ou ensino médio incompleto }\end{array}$ & 21 & 10,1 \\
\hline $\begin{array}{l}\text { Ensino médio ou superior } \\
\text { incompleto }\end{array}$ & 53 & 25,9 \\
\hline Superior completo & 76 & 37,1 \\
\hline \multicolumn{3}{|l|}{ Classe social } \\
\hline A2 & 16 & 7,8 \\
\hline B1/B2 & 67 & 32,7 \\
\hline $\mathrm{C} 1 / \mathrm{C} 2$ & 98 & 47,8 \\
\hline $\mathrm{D}$ & 24 & 11,7 \\
\hline \multicolumn{3}{|l|}{ Relação com o idoso } \\
\hline Filha(o) & 138 & 67,3 \\
\hline Neta(o) & 21 & 10,2 \\
\hline Cônjuge & 19 & 9,3 \\
\hline Nora/genro & 15 & 7,3 \\
\hline Irmã/irmão & 9 & 4,4 \\
\hline Outro & 3 & 1,5 \\
\hline \multicolumn{3}{|l|}{ Porte da cidade } \\
\hline Pequeno & 34 & 16,6 \\
\hline Médio & 118 & 57,6 \\
\hline Metrópole & 53 & 25,8 \\
\hline
\end{tabular}

\section{Análise de dados}

Os dados foram digitados em uma planilha dosoftware SPSS, seguida pela AFE do instrumento e sua consistência interna ( $\alpha$ de Cronbach). Todas as variáveis apresentaram uma distribuição normal, segundo a inspeção do número de modas, valores de curtose e assimetria e do teste de normalidade de Kolmogorov-Smirnov (Cozby, 2003; Marôco, 2014; Pasquali, 2015; Tornimbeni et al., 2008).

\section{Resultados}

Tendo em vista que a coleta de dados foi realizada por meio de entrevistas individuais, não houve problemas com dados omissos.

\section{Análise fatorial exploratória}

A amostra para a realização da análise fatorial exploratória incidiu sobre a totalidade dos cuidadores entrevistados $(n=205)$. O método de extração foi a fatoração de eixo principal, com método de rotação Varimax. Foram selecionados os itens com saturação superior a 0,40 e foram extraídos os fatores com eigenvalue superior a 1,5. A análise do scree plot sugeriu dois ou três fatores, apenas, diferente do modelo original do IHS-Del Prette, de cinco fatores. Em seguida, foram realizadas novas AFEs e comparados os modelos, mantendo os critérios anteriores, para verificar qual se adequava melhor à base conceitual de HS, ou seja, se a solução de dois ou a de três fatores. Em cada AFE, foram retirados os itens que não saturavam em nenhum dos fatores ou que apresentaram saturação em mais de um fator, até que nenhum item precisasse ser excluído.

$\mathrm{Na}$ estrutura com dois fatores, o valor de critério de Kaiser-Meyer-Olkin (KMO) foi de 0,877. Na primeira rodada, nove itens não saturaram em nenhum fator e foram retirados. Na segunda rodada, nenhum item precisou ser excluído. Ao final, a estrutura com 2 fatores apresentou $42,501 \%$ de variância total explicada. Para o Fator 1, o $\alpha$ de Cronbach foi 0,87; para o fator 2 foi 0,81 ; e 0,89 para a escala total.

$\mathrm{Na}$ estrutura com 3 fatores, o valor de $\mathrm{KMO}$ foi de 0,863 . Na primeira rodada de AFE, foram retirados quatro itens que não saturaram em nenhum fator e um que apresentou saturação em mais de um fator. $\mathrm{Na}$ rodada seguinte, um novo item foi retirado por não saturar em nenhum fator. $\mathrm{Na}$ terceira rodada, mais um item foi retirado por não saturar em nenhum fator. Na quarta rodada, nenhum item precisou ser excluído. Ao final, a estrutura com 3 fatores apresentou 45,273\% de variância total explicada e os seguintes alfas: fator $1, \alpha=0,87$; Fator 2, $\alpha=0,79$; fator $3, \alpha=0,60$; e escala global $\operatorname{com} \alpha=0,89$.

Percebe-se que as estruturas com dois e três fatores se mostraram estatisticamente adequadas. No entanto, após a comparação das soluções obtidas, a adequação ao modelo conceitual se mostrou mais satisfatória com a estrutura de três fatores. $\mathrm{O}$ fator 1 foi interpretado 
como espelhando uma dimensão de "expressividade afetiva", referindo-se às habilidades do cuidador para demonstrar afeto positivo em relação a outras pessoas e, em particular, ao idoso sob seus cuidados; o fator 2, "comunicação assertiva", referindo-se às habilidades do cuidador para lidar com interações difíceis que requerem enfrentamento e algum risco de reação indesejável por parte do outro, ou seja, com demandas de assertividade nas relações com o idoso sob seus cuidados e na relação com outras pessoas; e o fator 3, "busca de formação/informação", refere-se às habilidades do cuidador para buscar e disseminar informações relevantes para os cuidados do idoso. Observa-se, na Tabela 2, as correlações entre o escore geral e todos os fatores do presente instrumento, mostrando que todos os fatores se correlacionam moderadamente entre si e com o escore total.

Na Tabela 3, observa-se o modelo final com três fatores, após a AFE, e suas respectivas cargas de saturação.

\section{Precisão}

A precisão no presente estudo, para o fator 1 , "expressividade emocional", foi de $\alpha=0,87$; para o fator 2, "comunicação assertiva", $\alpha=0,79$; e para o fator 3, "busca de formação/informação", $\alpha=0,60$. Todos são considerados adequados para uma análise fatorial exploratória. $\mathrm{O} \alpha$ de Cronbach $(\alpha=0,89)$ geral foi excelente.

\section{Discussão}

O presente estudo teve como objetivo construir e validar um IHS para cuidadores familiares de idosos. Este objetivo foi abordado por meio de dois estudos: o primeiro para reportar evidências baseadas na análise do conteúdo ou domínio, examinadas durante o processo de construção e avaliação semântica dos itens e de conteúdo do instrumento; o segundo para obter evidências em relação à estrutura interna e precisão do instrumento.

Tabela 2

Correlações entre o escore total do Instrumento de Habilidades Sociais para Cuidadores familiares de Idosos e os escores nos três fatores

\begin{tabular}{lccc}
\hline & $\begin{array}{c}\text { Escore } \\
\text { total }\end{array}$ & $\begin{array}{c}\text { Expressivi- } \\
\text { dade afetiva }\end{array}$ & $\begin{array}{c}\text { Comunicação } \\
\text { assertiva }\end{array}$ \\
\hline $\begin{array}{l}\text { Expressividade } \\
\text { afetiva }\end{array}$ & $0,865^{*}$ & & \\
$\begin{array}{l}\text { Comunicação } \\
\text { assertiva }\end{array}$ & $0,842^{*}$ & $0,520^{*}$ & \\
$\begin{array}{l}\text { Busca de } \\
\text { formação/ } \\
\text { informação }\end{array}$ & $0,683^{*}$ & $0,483^{*}$ & $0,427^{*}$ \\
\hline Nota: ${ }^{*} p<0,01$. & & & \\
\end{tabular}

\section{Características psicométricas do instrumento}

O Estudo 1 mostrou que os pesquisadores das áreas de HS e psicometria concordaram que os itens propostos refletiam adequadamente os conceitos teóricos que deveriam representar. Além disso, com base em uma aplicação teste do instrumento, verificou-se que, após pequenos ajustes, pessoas da população-alvo, mesmo com baixa escolaridade, entenderam os itens e usaram corretamente a escala de pontuação. No entanto, o instrumento deve ser aplicado em

Tabela 3

Saturação dos Itens do Instrumento de Habilidades Sociais para Cuidadores familiares de Idosos, por fator

\begin{tabular}{|c|c|}
\hline Fator/itens & Saturação \\
\hline \multicolumn{2}{|l|}{ Fator 1: expressividade afetiva } \\
\hline $\begin{array}{l}\text { Item } 2 \text { - elogiar o idoso quando faz } \\
\text { algo bom }\end{array}$ & 0,790 \\
\hline Item 24 - demonstrar carinho & 0,762 \\
\hline Item 9 - animar o idoso & 0,747 \\
\hline $\begin{array}{l}\text { Item } 27 \text { - elogiar o idoso quando faz } \\
\text { algo difícil }\end{array}$ & 0,745 \\
\hline Item 22 - agradecer elogio do idoso & 0,683 \\
\hline Item 31 - se colocar no lugar do outro & 0,645 \\
\hline Item 4 - elogiar um familiar & 0,601 \\
\hline Item 1 - agradecer elogio de familiar & 0,569 \\
\hline $\begin{array}{l}\text { Item } 30 \text { - demonstrar insatisfação } \\
\text { para familiares }\end{array}$ & 0,482 \\
\hline Item 15 - controlar a irritação & 0,481 \\
\hline \multicolumn{2}{|l|}{ Fator 2: comunicação assertiva } \\
\hline Item 20 - aceitar ajuda de familiar & 0,697 \\
\hline Item 10 - pedir ajuda & 0,615 \\
\hline Item 8 - admitir um erro & 0,605 \\
\hline $\begin{array}{l}\text { Item } 14 \text { - agradecer colaboração } \\
\text { de familiares }\end{array}$ & 0,597 \\
\hline Item 12 - dizer o que sente & 0,585 \\
\hline Item 17 - conversar sobre dificuldades & 0,513 \\
\hline $\begin{array}{l}\text { Item } 6 \text { - mudar comportamento após } \\
\text { crítica construtiva }\end{array}$ & 0,536 \\
\hline Item 11 - concordar com crítica justa & 0,500 \\
\hline Item 18 - aceitar ajuda & 0,458 \\
\hline Item 25 - Dizer o que pensa & 0,446 \\
\hline \multicolumn{2}{|l|}{ Fator 3: busca de formação/informação } \\
\hline Item 3 - fazer perguntas a profissionais & 0,635 \\
\hline $\begin{array}{l}\text { Item } 29 \text { - fazer perguntas a } \\
\text { outros cuidadores }\end{array}$ & 0,579 \\
\hline Item 16 - pedir a opinião de outrem & 0,555 \\
\hline $\begin{array}{l}\text { Item } 19 \text { - interromper a interrupção } \\
\text { de outro }\end{array}$ & 0,546 \\
\hline
\end{tabular}


forma de entrevista para pessoas que não têm nenhuma escolaridade.

No Estudo 2, a avaliação das características psicométricas do instrumento foi iniciada por meio de uma AFE. Após a realização da AFE e a comparação dos dois modelos que apresentaram bons índices de ajustamento, os pesquisadores do presente estudo entraram em consenso de que o formato com melhor validade conceitual foi a estrutura de três fatores. Os pesquisadores também concordaram sobre a interpretação e nomeação de cada fator:

- Fator 1: expressividade afetiva;

- Fator 2: comunicação assertiva;

- Fator 3: busca de formação/informação.

No entanto, inicialmente esperava-se uma estrutura com cinco fatores, uma vez que o instrumento foi baseado em Del Prette e Del Prette (2001). Acredita-se então, que o fator "expressividade afetiva" contemplou o fator "autoafirmação na expressão de sentimentos positivos" de Del Prette e Del Prette (2001). Já o fator "comunicação assertiva" contemplou os fatores "enfrentamento e autoafirmação" e "autocontrole da agressividade", pois um cuidador que se mostra assertivo necessariamente precisa ter em seu repertório essas classes de comportamento (Del Prette \& Del Prette, 2013; Pinto et al., 2016). Por fim, o fator "busca de formação/informação" contemplou os fatores "conversação e desenvoltura social" e "autoexposição a desconhecidos e situações novas", uma vez que essas habilidades também são necessárias para que o cuidador tenha êxito em se formar e se informar para cuidar de seu parente idoso (Pinto et al., 2016). Esses fatores representam três classes de comportamentos essenciais e não sobrepostas de HS requeridas para cuidar de um idoso dependente, e estão de acordo com os achados da literatura, uma vez que são primordiais para o estabelecimento $\mathrm{e}$ a manutenção de relacionamentos de qualidade, tanto com o idoso cuidado quanto com outras pessoas que fazem parte da vida do cuidador (Muela, et al., 2001; Pinto \& Barham, 2014a; 2014b; Pinto et al., 2016).

Após esse refinamento do instrumento, a precisão (ou confiabilidade) dessa versão foi investigada por meio da análise da consistência interna das escalas. No presente estudo, a confiabilidade do IHS-CI se mostrou adequada tanto em sua forma global, como para cada fator. A confiabilidade do fator 1 foi considerada excelente, a do fator 2 foi considerada boa e a do fator 3 limítrofe; já a escala global foi considerada excelente (Pasquali, 2007; 2015; Tornimbeni et al., 2008). Quanto menor o número de itens, menor a probabilidade da medida de confiabilidade usada ( $\alpha$ de Cronbach) ser alta, porque múltiplos indicadores comportamentais (os itens) tendem a representar um construto latente (fator) melhor do que poucos indicadores (Marôco, 2014). Portanto, será importante preparar itens novos para o terceiro fator, para testar em estudos futuros, a fim de melhorar a precisão desse fator.

Além da necessidade de melhorar os índices de confiabilidade, outro resultado preocupante foi a ausência de itens com cargas fatoriais altas. $\mathrm{O}$ uso de um procedimento alternativo para extração dos fatores - por exemplo, oblimin, conforme Marôco (2014) —, resultaria em cargas fatoriais maiores, mas a desvantagem é que os fatores deixariam de ser independentes, o que seria um impedimento para calcular o escore total por meio da simples soma das pontuações de itens individuais. Fórmulas mais complexas poderiam ser usadas para ponderar as respostas de cada participante em cada item, mas um procedimento complicado para a apuração dos resultados poderia diminuir significativamente o uso do instrumento (Marôco, 2014; Pasquali, 2015). Apesar da necessidade de melhorar os índices de confiabilidade, pode-se concluir que essa versão inicial do IHS-CI, tanto em termos globais como em seus fatores, parece ser confiável e válida para avaliar as HS de cuidadores de idosos.

\section{Implicações para a atuação de psicólogos}

As possibilidades da aplicação de um instrumento de HS para cuidadores familiares de idosos são diversificadas. Com o envelhecimento populacional e a redução no tamanho das famílias, um instrumento de HS para cuidadores de idosos se torna relevante para a prática profissional em psicologia para:

1. identificar possíveis déficits e recursos no repertório de alguém que está começando a exercer esse papel, que podem orientar o planejamento de intervenções para superar esses déficits;

2. aferir o resultado de intervenções oferecidas por profissionais que visem aprimorar o repertório de HS dessa população, visando também ampliar a variedade de habilidades e a competência para lidar com as demandas desse contexto específico.

Além disso, por ser de fácil aplicação, esse instrumento poderá ser utilizado por profissionais em novas pesquisas, uma vez que informações sobre as HS de cuidadores de idosos ainda são escassas na literatura (Pinto et al., 2016).

Uma das principais limitações no processo de validação do instrumento diz respeito ao número de participantes. Para um instrumento com 31 itens, o número de participantes (205 cuidadores) superou o número mínimo recomendado para realizar sua AFE, seguindo as recomendações de Pasquali (1996; 1999). Não obstante, esse ainda é um número pequeno de respondentes considerando a intenção de validar o instrumento para uso em todo o Brasil. Assim, em estudos futuros, será necessário avaliar amostras multicêntricas mais representativas de cuidadores de idosos, residindo, por exemplo, em diferentes regiões do Brasil, e comparar os dados obtidos em cada região. Outra limitação 
foi o número restrito de participantes do sexo masculino $(n=25)$, quando comparado ao número de participantes do sexo feminino $(n=180)$. No entanto, essa diferença na proporção de cuidadores de cada sexo está condizente com achados de outros pesquisadores que indicam que a grande maioria dos cuidadores familiares de idosos é do sexo feminino (Gratão et al., 2013; Neri et al., 2012). Ainda em relação ao perfil dos cuidadores de idosos, é importante frisar que o instrumento é apropriado somente para cuidadores familiares de idosos, mas perante a presença cada vez maior de cuidadores formais, seria interessante e altamente relevante dispor também de um instrumento para avaliar cuidadores formais de idosos (Franzmann et al., 2014). Para a finalização da construção e da validação do presente instrumento, ainda são necessárias evidências adicionais e, principalmente, uma análise fatorial confirmatória para verificar se a estrutura fatorial encontrada para os participantes do presente estudo será confirmada ao se aplicar o instrumento em outros participantes sob os mesmos critérios de inclusão e exclusão. Outra etapa para a validação do instrumento é a verificação de seu relacionamento com outras variáveis já conhecidas na literatura, como menor índice de depressão, maior qualidade de vida, entre outras (Del Prette \& Del Prette, 2013). É importante ressaltar, ainda, que a literatura é carente nessa área específica de conhecimento, e que novos estudos são necessários, com o objetivo de amadurecer a compreensão da influência das HS sobre o bem-estar e a qualidade de vida de cuidadores de idosos, dos idosos cuidados e dos demais familiares e profissionais que tipicamente estão envolvidos nesse contexto.

\section{Referências}

ABEP (2013). Critério de Classificação Econômica Brasil. Associação Brasileira de Empresas de Pesquisa.

Aiken, L. R. (1980). Content validity and reliability of single items or questionnaires. Educational and. Psychological Measurement, 40(4), 955-959. doi: 10.1177/001316448004000419

Aiken, L. R. (1985). Three coefficients for analyzing the reliability and validity of ratings. Educational and Psychological Measurement, 45(1), 131-142. doi: 10.1177/0013164485451012

Barham, E. J., Pinto, F. N. F. R., Andrade, A. R., Lorenzini, M. F. J., \& Ferreira, C. R. (2015). Fundamentos e estratégias de intervenção para a promoção de saúde mental em cuidadores de idosos. Em S. G. Murta, C. Leandro-França, K. B. Santos \& L. Polejack (Orgs.), Prevenção e promoção em saúde mental: Fundamentos, planejamento e estratégias de intervenção (pp. 844-862). Novo Hamburgo, RS: Sinopsys.

Bender, R. S., \& Calvetti, P. U. (2015). Instrumentos de avaliação psicológica em habilidades sociais. Revista de Psicologia da IMED, 7(1), 4-14. Recuperado de https://seer.imed.edu.br/index.php/revistapsico/article/download/682/600

Cozby, P.C. (2003). Métodos de pesquisa em ciências do comportamento. São Paulo, SP: Atlas.

Del Prette, Z. A. P., \& Del Prette, A. (2001). Inventário de Habilidades Sociais (IHS-Del Prette): Manual de aplicação, apuração e interpretação. São Paulo, SP: Casa do Psicólogo.

Del Prette, Z. A. P., \& Del Prette, A. (2010). Avaliação de habilidades sociais: Bases conceituais, instrumentos e procedimentos. Em: A. Del Prette \& Z. A. P Del Prette (Orgs.), Psicologia das habilidades sociais: Diversidade teórica e suas implicações (pp. 189-231). Petrópolis, RJ: Vozes.

Del Prette, Z. A. P., \& Del Prette, A. (2013). Social Skills Inventory (SSI-Del-Prette): Characteristics and studies in Brazil. Em F. L. Osório (Org.), Social anxiety disorders: From theory to practice (pp. 49-62). Nova Iorque, NY: Nova Science Publishers.

Faleiros, D.A.M. (2009). Cuidadores de idosos com doença de Alzheimer: Efeitos de grupos psico-educacionais e suporte domiciliar individualizado. (Dissertação de Mestrado). Universidade Federal de São Carlos, São Carlos- SP.

Franzmann, J., Krause, K., Haberstroh, J., \& Pantel, J. (2014). Assessment of self-perceived social competencies of caregivers in dementia care: Development and psychometric testing of the SOKO dementia. GeroPsych, 27(2), 67-73. doi: 10.1024/1662-9647/a000103

Gratão, A. C. M., Talmelli, L. F. S., Figueiredo, L. C., Rosset, I., Freitas, C. P., \& Rodrigues, R. A. P. (2013). Dependência funcional de idosos e a sobrecarga do cuidador. Revista da Escola de Enfermagem -USP, 47(1), 137-44.

Li, R., Cooper, C., Bradley, J., Shulman, A., \& Ryan, G. (2012). Coping strategies and psychological morbidity in family carers of people with dementia: A systematic review and meta-analysis. Journal of Affective Disorders, 139(1), 1-11. doi: 10.1016/j.jad.2011.05.055

Lin, W. F., Chen, H. C, \& Li, T. S. (2013). Adult children's caregiver burden and depression: The moderating roles of parent-child relationship satisfaction and feedback from others. Journal of Happiness Studies, 14(2), 673-687. doi: 10.1007/s10902-012-9348-0

Lorenzini, M. F. J. (2014). Trabalhadores que Cuidam de Parentes Idosos: Esforços e Necessidades na Conciliação de Responsabilidades Profissionais e Familiares (Trabalho de conclusão de curso em Psicologia). Universidade Federal de São Carlos, São Carlos, SP.

Marôco, J. (2014). Análise estatística com o SPSS statistics. Pêro Pinheiro, Portugal: Report Number.

Muela, J. A., Torres, C. J., \& Peláez, E. M. (2001). La evaluación de la asertividad como predictor de carga en cuidadores de enfermos de Alzheimer. Revista Española de Geriatria y Gerontologia, 36(1), 41-45.

Neri, A. L., Yassuda, M. S., Fortes- Burgos, A. C. G., Mantovani, E. P., Arbex, F. S., Torres, S. V. S., Perracini, M. R., \& Guariento, M. E. (2012). Relationships between gender, age, family conditions, physical and mental health, and social isolation of elderly caregivers. International Psychogeriatrics, 24(3), 472-483. doi: 10.1017/S1041610211001700

Novelli, M. M. P. C., Nitrini, R., \& Caramelli, P. (2010). Cuidadores de idosos com demência: Perfil sociodemográfico e impacto diário. Revista de Terapia Ocupacional da Universidade de São Paulo, 21(2), 139-147. Recuperado de www.revistas.usp.br/rto/article/ download/14097/15915 
Pasquali, L. (1996). Teoria e métodos de medida em ciências do comportamento. Brasília: Laboratório de Pesquisa em Avaliação e Medida da Universidade de Brasília.

Pasquali, L. (1999). Instrumentos psicológicos: Manual prático de elaboração. Brasília: Labpam/IBAPP.

Pasquali, L. (2007). Validade dos testes psicológicos: Será possível reencontrar o caminho? Psicologia: Teoria e pesquisa, $23,99-107$.

Pasquali, L. (2015). Delineamento de pesquisa em ciência: Volume 2. São Paulo, SP: Vetor.

Pereira, M. G., \& Carvalho H. (2012). Qualidade vida, sobrecarga, suporte social, ajustamento conjugal e morbidade psicológica em cuidadores de idosos com dependência funcional. Temas em Psicologia, 20(2), 369 - 383. doi: 10.9788/TP2012.2-07

Pinto, F. N. F. R, Barham, E. J., \& Albuquerque, P. P. (2013). Idosos vítimas de violência: Fatores sociodemográficos e subsídios para futuras intervenções. Revista Estudos e Pesquisa em Psicologia. 13(3), 1159-1181. Recuperado de http://www.e-publicacoes.uerj.br/index.php/ revispsi/article/view/8610/6579

Pinto, F. N. F. R., \& Barham, E. J. (2014a). Bem estar psicológico: Comparação entre cuidadores de idosos com e sem demência. Psicologia, Saúde \& Doenças, 15(3), 635-655. doi: 10.15309/14psd150307

Pinto, F. N. F. R., \& Barham, E. J. (2014b). Habilidades sociais e estratégias de enfrentamento de estresse: Relação com indicadores de bem-estar psicológico em cuidadores de idosos de alta dependência. Revista Brasileira de Geriatria e Gerontologia, 15(3), 525-539. doi: 10.1590/1809-9823.2014.13043

Pinto, F. N. F. R., Barham, E. J., \& Del Prette, Z. A. P. (2016). Interpersonal conflicts among family caregivers of the elderly: The importance of social skills. Paidéia (Ribeirão Preto), 26(64), 161-170. doi:10.1590/1982-43272664201605

Robinson, K. M. (1988). A social skills training program for adult caregivers. Advances in Nursing Science, 10(20), 59-72.

Robinson, K. M. (1990). The relationships between social skills, social support, self-esteem and burden in adult caregivers. Journal of Advanced Nursing, 15(7), 788-795.

Robinson, K. M., \& Yates, K. (1994). Effects of Two Caregiver-Training Programs on Burden and Attitude Toward Help. Archives of Psychiatric Nursing, 8(5), 312-319.

Tomomitsu, M. R. S. V., Perracini, M. R., \& Neri, A. L. (2014). Fatores associados à satisfação com a vida em idosos cuidadores e não cuidadores. Ciência E Saúde Coletiva, 19(8), 3429-3440. doi: 10.1590/1413-81232014198.13952013

Tornimbeni, S., Pérez, E., \& Olaz, F. (2008). Introducción a la psicometria. Buenos Aires: Paidós.

Wang, X. R., Robinson, K. M, \& Carter-Harris, L. (2014). Prevalence of chronic illnesses and characteristics of chronically ill informal caregivers of persons with dementia. Age and Ageing, 43(1), 137-141. doi: 10.1093/ageing/aft142

recebido em julho de 2016 reformulado em outubro de 2016 aprovado em novembro de 2016

\section{Sobre os autores}

Francine Náthalie Ferraresi Rodrigues Queluzé Psicóloga, Mestre e Doutora em Psicologia pela Universidade federal de São Carlos e Pós-doutoranda em Avaliação Psicológica pela Universidade São Francisco. Trabalha com o tema relacionamento cuidador-idoso, com enfoque nas habilidades sociais e estratégias de enfrentamento de estresse.

Elizabeth Joan Barham é Doutora e Professora do Departamento de Psicologia da Universidade Federal de São Carlos, trabalha com envelhecimento e equilibro trabalho-família.

Zilda Aparecida Pereira Del Prette é Doutora e Professora do Departamento de Psicologia da Universidade Federal de São Carlos, trabalha com habilidades sociais e no desenvolvimento de instrumentos na área.

Anne Marie Germaine Victoria Fontaine é Doutora e Professora do Departamento de Psicologia da Universidade do Porto, trabalha com relações intergeracionais e psicometria.

Fabian Orlando Olaz é Doutor e Professor do Departamento de Psicologia da Universidade Nacional de Córdoba, trabalha com o desenvolvimento de instrumentos, habilidades sociais e terapia de aceitação e compromisso. 